EPJ Web of Conferences 32, 02013 (2012)

DOI: $10.1051 /$ epjconf/20123202013

(C) Owned by the authors, published by EDP Sciences, 2012

\title{
Monitoring millimeter wave stray radiation during ECRH operation at ASDEX Upgrade
}

\author{
M. Schubert ${ }^{\mathrm{a}}$, F. Honecker, F. Monaco, D. Schmid-Lorch ${ }^{\mathrm{b}}$, H. Schütz, J. Stober, D. Wagner and the \\ ASDEX Upgrade Team
}

Max-Planck-Institut für Plasmaphysik, EURATOM Association, Garching, Germany

\begin{abstract}
Due to imperfection of the single path absorption, ECRH at ASDEX Upgrade (AUG) is always accompanied by stray radiation in the vacuum vessel. New ECRH scenarios with $\mathrm{O} 2$ and $\mathrm{X} 3$ heating schemes extend the operational space, but they have also the potential to increase the level of stray radiation. There are hazards for invessel components. Damage on electric cables has already been encountered. It is therefore necessary to monitor and control the ECRH with respect to the stray radiation level. At AUG a system of Sniffer antennas equipped with microwave detection diodes is installed. The system is part of the ECRH interlock circuit. We notice, however, that during plasma operation the variations of the Sniffer antenna signal are very large. In laboratory measurements we see variations of up to $20 \mathrm{~dB}$ in the directional sensitivity and we conclude that an interference pattern is formed inside the copper sphere of the antenna. When ECRH is in plasma operation at AUG, the plasma is acting as a phase and mode mixer for the millimeter waves and thus the interference pattern inside the sphere changes with the characteristic time of the plasma dynamics. In order to overcome the difficulty of a calibrated measurement of the average stray radiation level, we installed bolometer and pyroelectric detectors, which intrinsically average over interference structures due to their large active area. The bolometer provides a robust calibration but with moderate temporal resolution. The pyroelectric detector provides high sensitivity and a good temporal resolution, but it raises issues of possible signal drifts in long pulses.
\end{abstract}

\section{Introduction}

The electron cyclotron heating system at ASDEX Upgrade (AUG) consists of seven units, which routinely operate at $f=140 \mathrm{GHz}\left(\lambda_{0}=2.1 \mathrm{~mm}\right)$, three units can also operate at $105 \mathrm{GHz}$. Up to $4 \mathrm{MW}$ of microwave power are launched at four different toroidal positions (Figure 1). A small fraction of this power can be found elsewhere in the vacuum vessel, whenever the absorption in the plasma is incomplete. This can happen due to a variety of geometrical effects, where for example the variation of the refractive index in the plasma gives rise to a reflection. Heating schemes using $\mathrm{O} 2$ or X3 inherently suffer from reduced single pass absorption [1]. We refer to the non-absorbed ECRH power as millimeter wave stray radiation. If its intensity is too high, it may damage in-vessel components.

Sniffer antennas [2] equipped with beam lead detection diodes have been installed close to each launcher in order to monitor the stray radiation. The idea of such a detector is to have a multi-modal and polarization independent antenna with broad directional sensitivity [2]. We used the antennas in order to characterize the relative stray radiation level in various AUG discharges as a function of the

${ }^{a}$ e-mail: Martin.Schubert@ipp.mpg.de

${ }^{b}$ actual affiliation: Universität Stuttgart, 3.Physikalisches Institut, Pfaffenwaldring 57, 70569 Stuttgart, Germany 
toroidal distance to the launcher position (cf. Figure 2). If the X2 mode is launched perpendicular to the magnetic field, the stray radiation decreases by a factor of 100 within $\Delta \varphi=100^{\circ}$ in toroidal direction [3]. Near the launcher position a calibration factor of approx. $0.235 \mathrm{~W} / \mathrm{cm}^{2}$ per $\mathrm{MW}$ of the launched Gyrotron power has been measured in a port $1.5 \mathrm{~m}$ outside the separatrix [3]. This factor, however, is put into question by two observations: (i) The directional sensitivity of the Sniffer antenna shows large variations of up to $20 \mathrm{~dB}$ at $f=140 \mathrm{GHz}$. This is the result of a laboratory measurement and will be shown in section 2.1. (ii) The mean deviation of the experimentally obtained signal from the Sniffer antenna during plasma operation is of the same order as the average value. The corresponding results from AUG are shown in section 3.

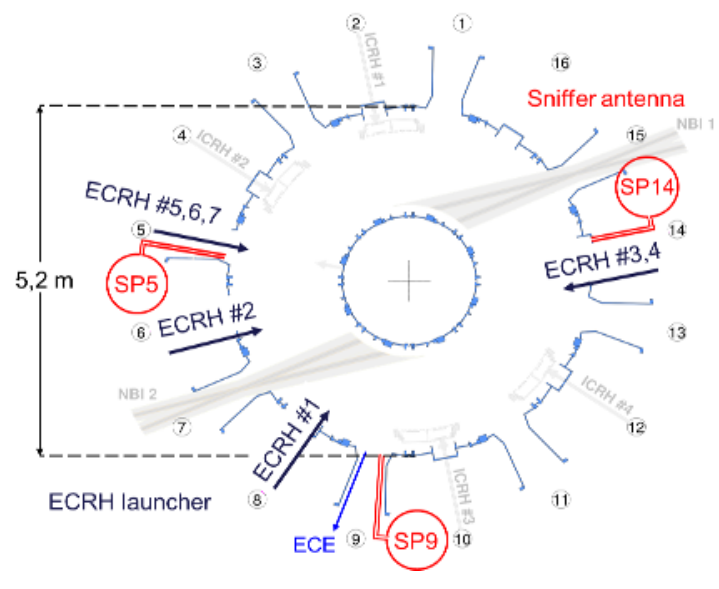

Fig. 1 Schematic top view of ASDEX Upgrade: Location of the ECRH launchers and of the Sniffer antennas (SP), which are used as stray radiation monitors.

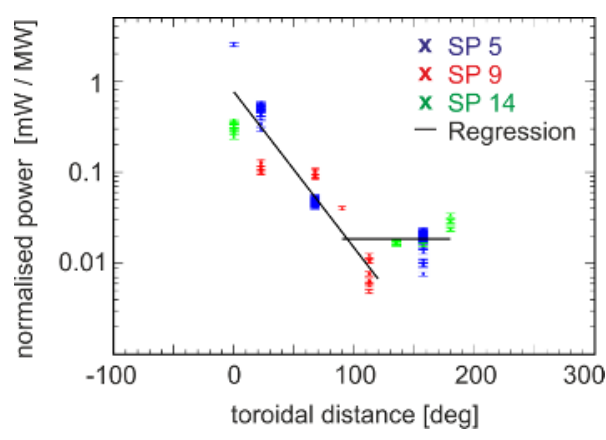

Fig. 2 Power measured by the detector of the Sniffer probe normalized to $1 \mathrm{MW}$ of launched Gyrotron power, function of the toroidal distance to the launcher position [3]. Data corresponds to perpendicular launch and X2 Mode.

We report on our continued investigation [4] of the properties of the Sniffer antenna in section 2 and we collect evidence, that the deviations in the directional sensitivity and in the plasma signals are due to interference. With the aim to obtain better accuracy of the measured stray radiation intensity, we installed a resistive bolometer and a pyroelectric detector. Both have a large enough detector area to intrinsically average over interference structures. We compare a simple model with the experimentally observed variations in section 2.2 .

\section{Detection methods}

\subsection{Sniffer antenna}

We show the scheme of a Sniffer antenna in Figure 3. Millimeter wave radiation enters the copper sphere through an oversized waveguide. The function of the sphere is to allow for multiple reflections, so that the intensity inside the sphere is supposed to be homogeneous and contains all polarizations [2]. The sphere has a small aperture with a D-band waveguide flange, where a single beam lead diode is mounted and serves as a millimeter wave power detector.

In laboratory measurements [3] we use an Extended Interaction Oscillator (EIO) as $140 \mathrm{GHz}$ power source and find, that the directional sensitivity of this system is not smooth (Figure 4). Indeed it contains several narrow intervals, where the sensitivity drops by $-20 \mathrm{~dB}$. When the measurement is repeated after some minutes of waiting time, the intervals are found at different positions. It is known that the EIO frequency drifts on a slow timescale due to thermal effects. In recent experiments [4] we succeeded to stabilize the EIO frequency by improving the temperature control in the cooling system. We find now, that the sensitivity pattern can be reproduced even after a long waiting time (Figure 5), if the frequency is stable within $\Delta f \leq 1 \mathrm{MHz}$. We conclude therefore that the intensity 


\section{EC-17 Workshop}

inside the sphere is not homogeneous, probably because the number of reflections of the order of 1000 is not large enough. This is determined by the ohmic losses and by the ratio of the waveguide opening to the surface of the sphere. In fact a discrete spectrum of spherical eigenmodes is excited by the incident beam. The spectrum depends on the frequency and on the angle of incidence and it only contains a limited number of modes, so that an interference pattern with fine structures is formed on the surface of the sphere. Since the beam lead detector diode is small compared to the wavelength, the interference structures are reproduced in the measurement data.

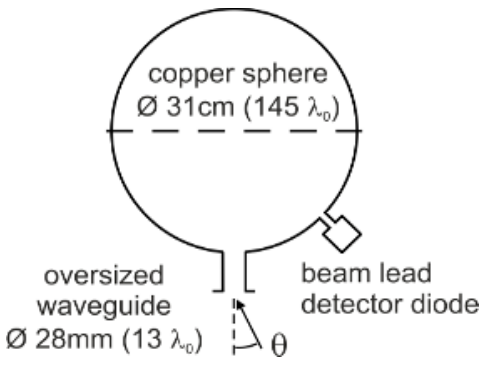

Fig. 3 Scheme of the Sniffer antenna. Two halves are connected with an equatorial flange and form a hollow sphere. The lower half has a circular opening $(\varnothing 28 \mathrm{~mm})$ for the oversized waveguide and a small aperture $(\varnothing 5 \mathrm{~mm})$ for the D-band waveguide of the beam lead diode.

\subsection{Bolometer detector}

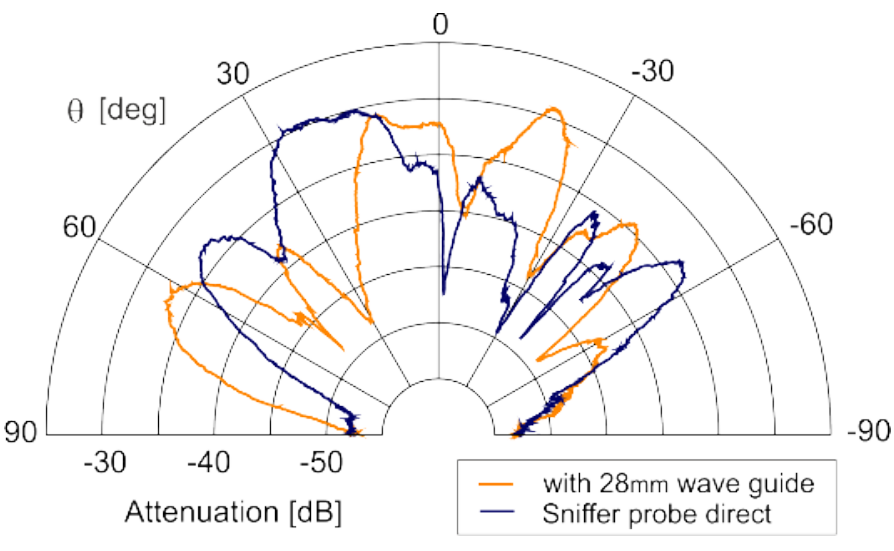

Fig. 4 Measured directional sensitivity of the Sniffer antenna [3]. Due to a frequency drift of the $140 \mathrm{GHz}$ source, the two measurements correspond to slightly different source frequencies. The length of the oversized waveguide was also changed.

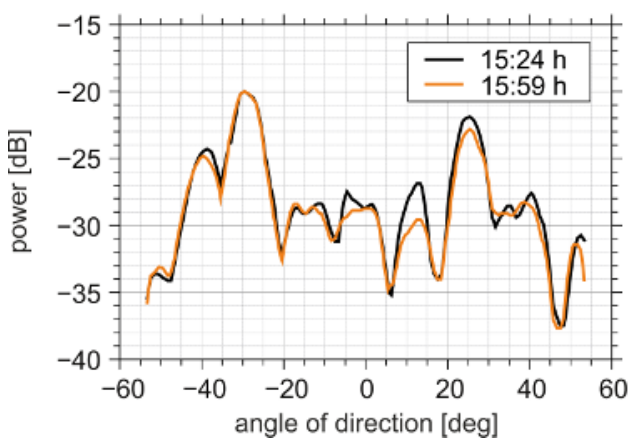

Fig. 5 Measured directional sensitivity of the Sniffer antenna with stabilization of the source frequency [4]. Variations of up to $20 \mathrm{~dB}$ are still present, but they can be reproduced at the same angle of incidence even after a long waiting time.

Since we have to cope with interference, our next step was to test detectors where the detector area is large compared to the size of interference structures. Given the detector signal is proportional to the surface average of the intensity, then the directional sensitivity should be smooth. We noticed, that bolometers which measure broad-band plasma radiation at AUG (in particular in the visible light, VUV and soft X-ray range), are also sensitive to millimeter waves. This was also observed on the DIII-D tokamak [5]. Bolometers contain a thermally decoupled component, which will heat up due to absorption of radiation when exposed. The temperature of this absorbing component is monitored by a thermometer, a temperature dependent metallic resistor in our case. Once the radiation is stopped, the absorber returns to the ambient temperature with a characteristic time constant, that depends on the heat capacity of the absorber and on the thermal insulation. 
We installed an ITER prototype bolometer [6] in a laboratory set-up together with the EIO source, operating at $f=140 \mathrm{GHz}$. This type of bolometer (see Figure 6 ) has a detector area of $\approx 6 \mathrm{~mm}^{2}$ per channel, larger than the estimated size of interference structures of the order $\left(\lambda_{0} / 2\right)^{2} \approx 1 \mathrm{~mm}^{2}$. The thermal time constant of approx. $150 \mathrm{~ms}$ is considered fast enough for our application. We measured the directional sensitivity in both angular directions and with two different polarizations of the millimeter wave (Figure 7). The characteristics are very smooth, as expected. The angular range of acceptance is slightly smaller than that of the Sniffer probe. This should be due to the narrow design of the ITER prototype bolometer aperture, which we did not change for simplicity. There is room for optimization by a new design of the aperture.

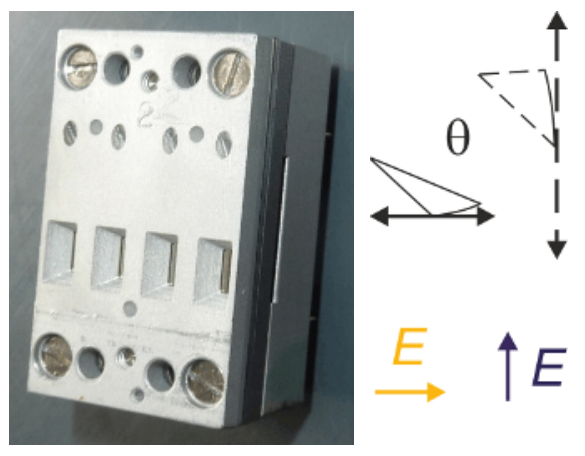

Fig. 6 ITER prototype bolometer [6] and definition of directions for the measurement of the directional sensitivity. The four rectangular openings in the photo on the left correspond to the four measurement channels.

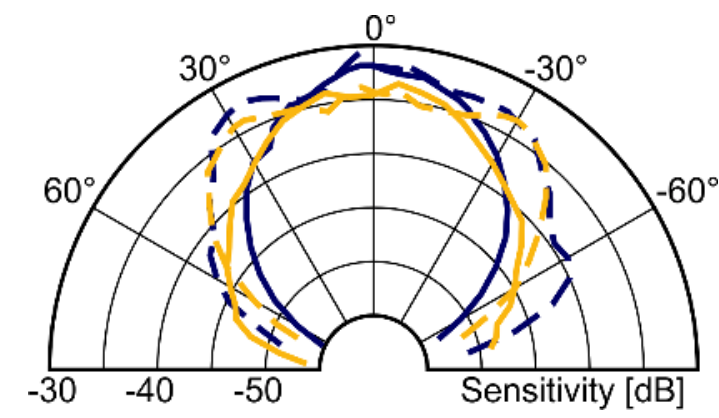

Fig. 7 Measured directional sensitivity of the Bolometer. Dashed lines for the vertical scans, solid lines for the horizontal scans, as defined in Figure 6. The dark lines correspond to the vertical polarization of the millimeter wave (i.e. electric field vector parallel to the long side of the rectangular aperture). In this case the sensitivity for $\theta=0^{\circ}$ is slightly higher than for the horizontal polarization.

We measured an overall absorption factor of the bolometer of $0.19 \%$ in accordance with the theoretical value for the platinum surface [4]. The theoretical value depends on the electrical conductivity of the surface material and on the wave frequency.

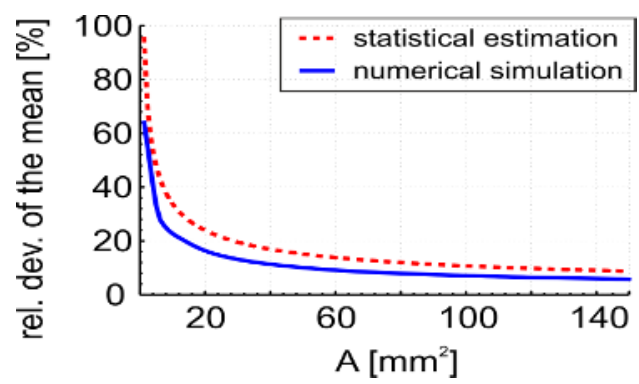

Fig. 8 Statistical and numerical estimation of the relative deviation of the measurement from the mean intensity [4]. The parameter on the $\mathrm{x}$-Axis is the detector area $A$. We assume a given frequency $f=140 \mathrm{GHz}$ and a $2 \mathrm{D}$ standing wave pattern with a $\lambda_{0} / 2$ distance between adjacent nodes.

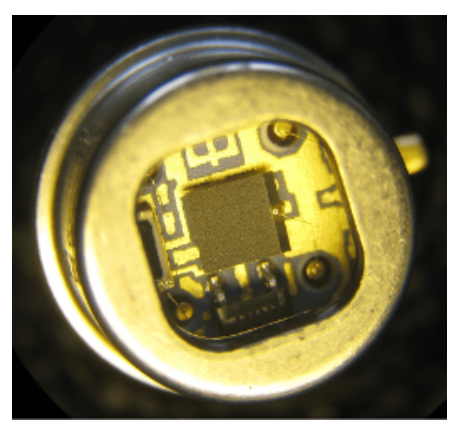

Fig. 9 Pyroelectric detector LIE-382. The diameter of the outer casing is approx. $8 \mathrm{~mm}$.

We observed also, that single channel signals varied within $40 \%$. This has been compared [4] to a very simple statistical estimation where we evaluate the square root of the ratio of the detector area $A$ to $\left(\lambda_{0} / 2\right)^{2}$ for the interference structure size, as a function of $A$ (Figure 8). A cross-check has been done [4] with a numerical simulation where the total intensity in a 2D standing wave pattern for a set of different wave phases has been calculated. Both estimations are in reasonable agreement with our experimental results. If the signal is averaged over all four bolometer channels (total area $24 \mathrm{~mm}^{2}$ ), we find variations of up to $30 \%$, also compatible with the statistical and the numerical approach. 


\subsection{Pyroelectric detector}

Similar to the bolometer, the pyroelectric detector contains an absorber unit which heats up when exposed to radiation. Conversion of the absorber temperature into an electrical signal is done by the pyroelectric effect, which generates an electrical charge as a function of the temperature gradient. We used an InfraTec LIE-382 type (Figure 9), which has a similar aperture area as one bolometer channel and also a similar thermal time constant of approx. $150 \mathrm{~ms}$. We focussed on the capability of this detector to monitor fast changes of the stray radiation intensity in plasma experiments and skipped both the calibration and the laboratory measurement of the directional sensitivity.

\section{Measurement Results at ASDEX Upgrade}

The Sniffer antenna is connected to the AUG vacuum vessel by an oversized waveguide. Bolometer and pyroelectric detector are installed at two miter bends (Figure 10) of the transmission line of SP5 (cf. Figure 1). Laboratory measurements [4] showed that the additional damping of mm-waves introduced by these components can be neglected. The air in the waveguide suppresses VUV radiation and the first miter bend prevents direct exposure to visible light from the plasma.
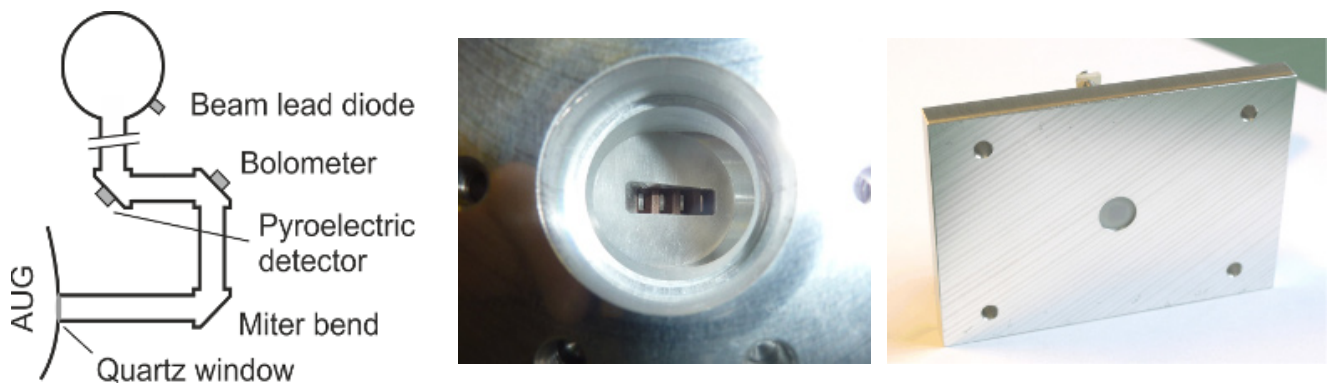

Fig. 10 Set-up of Sniffer antenna (with beam lead diode detector), bolometer and pyroelectric detector at AUG. The four channel bolometer (middle) and the pyroelectric detector (right) are installed at two modified miter bends of the oversized waveguide.

The capability of the detectors is demonstrated in two different AUG discharges. Figure 11 shows the pulse response of the three detectors (Sniffer, bolometer, pyroelectric) to a $20 \mathrm{~ms}$ ECRH pulse in $\mathrm{O} 2$ mode in a stationary plasma. Due to reduced single pass absorption, the stray radiation level is comparatively high. Figure 11 shows also that the detectors are efficiently shielded from other types of plasma radiation. The differentiating character of the pyroelectric and the integrating character of the bolometer raw signal are visible.

The stray radiation from a plasma experiment with stationary ECRH X2 heating is shown in Figure 12. Due to the differentiating character of the pyroelectric detector, the raw signal returns to the zero level in the stationary phase. The stray radiation power has been evaluated by adding the integral. There remains an offset in the power signal after switching off the ECRH. It is supposed that this is due to long-term thermal effects in the pyroelectric detector.

The Sniffer antenna signal has very large deviations which are comparable to the signal amplitude. We suppose that the AUG plasma dynamics acts as phase and mode mixer for the stray radiation, which continuously changes the mode spectrum in the Sniffer antenna. At least part of the signal deviations are supposed to be due to interference effects. For real-time processing of this signal, a low pass filter is inevitable, at the expense of temporal resolution.

Due to the integrating character of the bolometer, the bolometer signal is smooth. Part of the stray radiation dynamics is intrinsically averaged in time and cannot be reconstructed. Using the calibration of the bolometer that was done in the laboratory [4], we can evaluate a stationary stray radiation intensity of $5 \mathrm{~W} / \mathrm{cm}^{2}$ in the AUG vacuum vessel at the entrance of the quartz window. This calculation has been done according to [6] and taking into account the time derivative of the signal. 
The result is shown qualitatively in Figure 12 (bottom). When we normalize this result to the total ECRH power of 2.3 MW we obtain a stray radiation intensity which is significantly higher than what has been measured before (section 1 and [3]) by the Sniffer antenna alone. This could be an effect of the different range of directional acceptance of the two detection systems in combination with a nonisotropic stray radiation. Further investigation is needed to clarify this point.

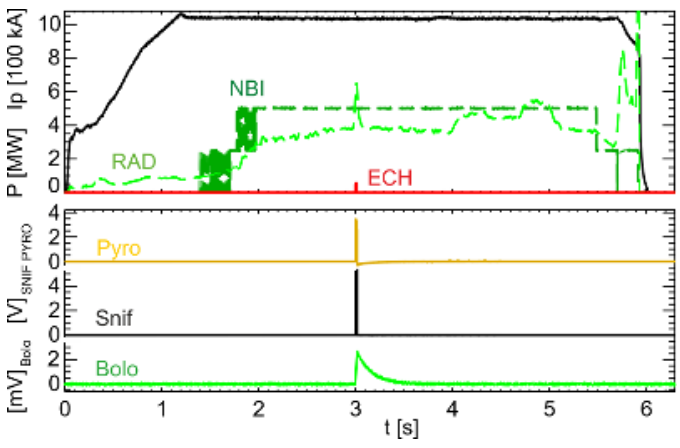

Fig. 11 Plasma experiment \#27700 at AUG. Top: plasma current, plasma radiation (RAD), neutral beam (NBI) and ECRH heating power. Bottom: raw signals of the pyroelectric detector, the Sniffer antenna (beam lead diode) and the bolometer.

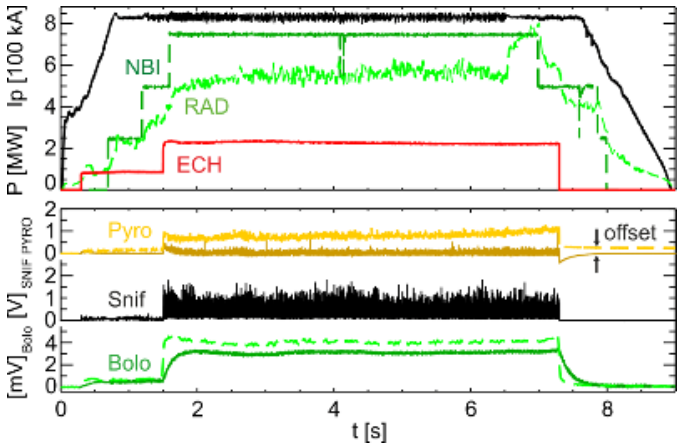

Fig. 12 Same signals as in Figure 11 for AUG experiment \#27577. Stationary ECRH X2 heating. Bottom: evaluation of radiation power from the raw pyroelectric and bolometer signals is indicated qualitatively in light colour and a dashed line.

\section{Summary and Conclusions}

Smoothed $(30 \mathrm{~ms})$ Sniffer antenna signals are routinely included in the AUG ECRH interlock system. They are robust and do not need numerical integration or differentiation. The calibration of stray radiation intensity, however, remains unclear. We showed that the effect of interference is always present. From this it can be concluded that the copper sphere of the Sniffer antenna is not essential and that the beam lead diode directly mounted at the quartz window and with adequate attenuators could have similar properties. The bolometer provides a very stable signal with a robust laboratory calibration to stray radiation intensity. Its acceptance angle is limited by the aperture, which needs a new design. The set-up at AUG in a miter bend provides good shielding from other types of radiation. The time resolution seems not to be sufficient for the needs of an interlock system that has to react within $<1 \mathrm{~ms}$. Pyroelectric detectors could be a good compromise, but the issue of a possible signal drift in long pulses ( $>>10 \mathrm{~s})$ needs to be solved.

\section{References}

1. H. Höhnle et al., Nucl. Fusion 51, 083013 (2011).

2. F. Gandini et al., Fusion Engineering and Design 56-57, 975 (2001).

3. D. Schmid-Lorch, Mikrowellen-Streustrahlung am Tokamak ASDEX Upgrade, diploma thesis Universität Ulm (2010).

4. F. Honecker, Neue Methoden zur Messung der Streustrahlung bei Millimeterwellen-Heizung (ECRH) an ASDEX Upgrade, diploma thesis Technische Universität München (2012).

5. J. Lohr et al., Proc. $16^{\text {th }}$ Workshop on Electron Cyclotron Emission and Electron Cyclotron Resonance Heating (EC-16), Ronald Prater Ed, World Scientific, Singapore, 339 (2011).

6. L. Giannone et al., Plasma Phys. Control. Fusion 47, 2123 (2005). 